\title{
An end-to-end workflow for multiplexed image processing and analysis
}

\author{
Jonas Windhager ${ }^{1,2,3,}{ }^{*}$, Bernd Bodenmiller ${ }^{1,2}$, and Nils Eling ${ }^{1,2,}{ }^{*}$ \\ ${ }^{1}$ Department of Quantitative Biomedicine, University of Zurich, Zurich, Switzerland \\ ${ }^{2}$ Institute for Molecular Health Sciences, ETH Zurich, Zurich, Switzerland \\ ${ }^{3}$ Life Science Zurich Graduate School, ETH Zurich and University of Zurich, Zurich, Switzerland \\ *Correspondence: jonas.windhager@uzh.ch, nils.eling@uzh.ch
}

Simultaneous profiling of the spatial distributions of multiple biological molecules at single-cell resolution has recently been enabled by the development of highly multiplexed imaging technologies. Extracting and analyzing biologically relevant information contained in complex imaging data requires the use of a diverse set of computational tools and algorithms. Here, we report the development of a userfriendly, customizable, and interoperable workflow for processing and analyzing data generated by highly multiplexed imaging technologies. The steinbock framework supports image pre-processing, segmentation, feature extraction, and standardized data export. Each step is performed in a reproducible fashion. The imcRtools $\mathrm{R} /$ Bioconductor package forms the bridge between image processing and single-cell analysis by directly importing data generated by steinbock. The package further supports spatial data analysis and integrates with tools developed within the Bioconductor project. Together, the tools described in this workflow facilitate analyses of multiplexed imaging raw data at the single-cell and spatial level. 


\section{Introduction}

Highly multiplexed imaging enables the simultaneous detection of tens of biological molecules (e.g., proteins, RNA), also referred to as "markers", in their spatial tissue context. Recently established multiplexed imaging technologies rely on cyclic staining with immunofluorescently tagged antibodies [1, 2], or multiplexed staining with oligonucleotide-tagged [3, 4] or metaltagged antibodies [5, 6]. The acquired data are commonly stored as multi-channel images, where each pixel encodes the abundances of all acquired markers at a specific position in the tissue. After data acquisition, bioimage processing and segmentation are conducted to extract data for downstream analysis. End-to-end multiplexed image analysis currently requires a diverse set of computational tools and complex analysis scripts.

Quantitative analysis of biological entities captured by multiplexed imaging requires processing of multi-channel images. This involves image extraction and pre-processing, image segmentation, and the quantification of biological objects such as cells. Multiplexed image segmentation is often performed by first classifying image pixels as nuclear, cytoplasmic or background (e.g., using software such as Ilastik [7]), followed by identifying and segmenting cells based on the resulting pixel-level class probabilities (e.g., using software such as CellProfiler [8]). Several pipelines have been developed for multi-channel image processing using pixel classification-based segmentation approaches, including the IMC Segmentation Pipeline [9], imcyto [10], and MCMICRO [11].

Classification-based segmentation approaches require the training of pixel classifiers (i.e., manual annotation of images), a process that is specific to the acquired markers. To enable applicability across marker panels, the dimensionality of input images can be reduced by aggregating selected channels. For example, a two-channel nuclear/cytoplasm image can be constructed from a multichannel image by averaging all nuclear and all cytoplasmic channels. Such channel-aggregated images can then be used to train panel-agnostic pixel classifiers to imitate classifiers previously trained on specific sets of markers [12], or to directly apply generalist cell segmentation algorithms 13 15. The latter methodologies include deep learning-enabled algorithms achieving human-level performance across various tissue types and imaging platforms [15.

In recent years, graphical user interface (GUI) software specialized for multiplexed imaging platforms have been developed to analyze cells with regards to their spatial location 16 19]. These tools are user-friendly and allow joint visualization of different data representations (e.g., images, single-cell features), but they often have little interoperability and are difficult to extend. Alternatively, after multi-channel image processing, the extracted tabular data can be analyzed using common programming languages such as $\mathrm{R}$ and Python. The squidpy Python package was developed recently to analyze spatial molecular data [20], and giotto performs similar analyses in $\mathrm{R} 21$. In contrast to stand-alone tools, the Bioconductor project offers interoperability among diverse analysis packages by relying on standardized data classes [22]. An example of such is the SingleCellExperiment class that supports general single-cell analyses including clustering of cells and dimensionality reduction [23 25], spatial clustering [26], and visualization of multiplexed imaging data 27]. 
bioRxiv preprint doi: https://doi.org/10.1101/2021.11.12468357; this version posted November $13,2021$. The copyright holder for this preprint (which was not certified by peer review) is the author/funder, who has granted bioRxiv a license to display the preprint in perpetuity. It is made available under aCC-BY 4.0 International license.

\section{Results}

Here, we present a modular and interoperable computational workflow to process and analyze multiplexed imaging data. The steinbock framework facilitates multi-channel image processing including raw data pre-processing, image segmentation, and feature extraction. Data generated by steinbock can be directly read using the imcRtools $\mathrm{R} /$ Bioconductor package, which also provides functionality for data visualization and spatial analysis (Figure 1).

\section{Multiplexed imaging data}

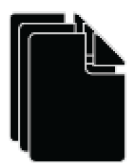

.tiff, .ome.tiff, .mcd, etc.

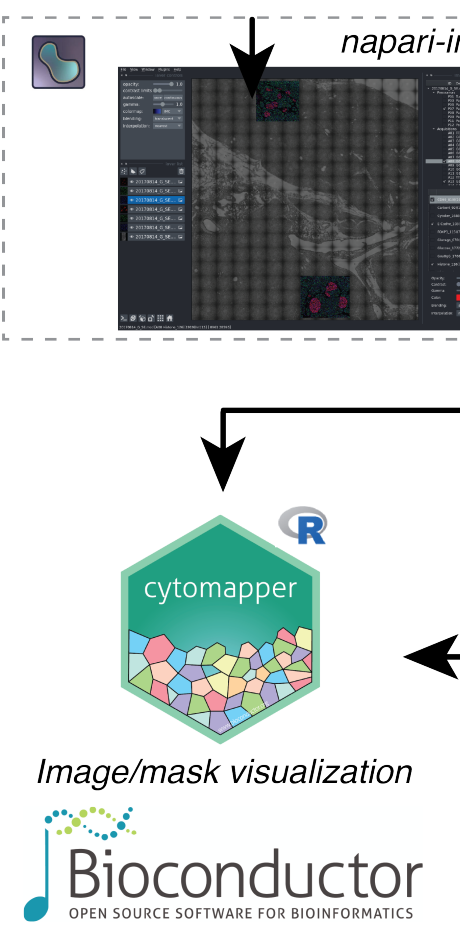

Single-cell analysis
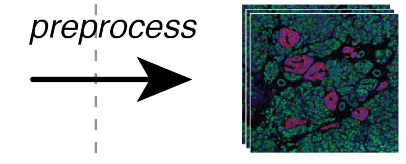
segr
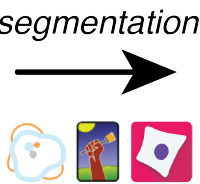
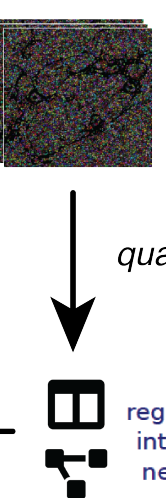

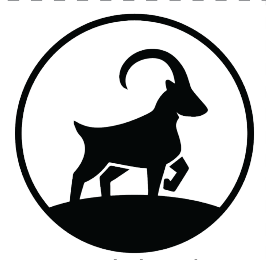

steinbock quantification

images, masks

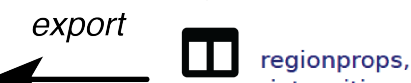
regionprops, intensities, neighbors

\section{measurements, graphs, etc.}

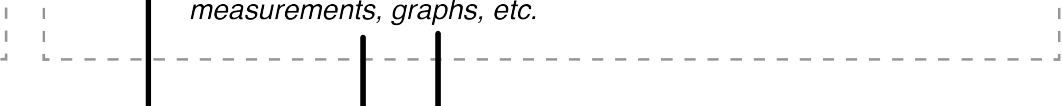

Figure 1: Overview of the multiplexed image processing and analysis workflow. Raw image data can be interactively visualized using napari plugins such as napari-imc for IMC to assess data quality and for exploratory visualization. The steinbock framework performs image pre-processing, cell segmentation and single-cell data extraction using established approaches and standardized file formats. Data can be read into $\mathrm{R}$ using the imcRtools package, which supports spatial visualization and analysis. Storing the data in SingleCellExperiment or SpatialExperiment objects, imcRtools integrates with a variety of data analysis tools of the Bioconductor project such as cytomapper [27]. Data can also be exported from steinbock as anndata objects to facilitate analysis in Python, e.g., using squidpy 20].

The presented workflow is user-friendly, customizable and reproducible, and integrates with a variety of downstream analysis strategies by employing standardized data formats. The tools included in this workflow support processing and analysis of data generated by a range of mul- 
tiplexed imaging technologies. For demonstration purposes, we present data from imaging mass cytometry (IMC), which relies on tissue staining with metal-labelled antibodies to simultaneously measure the spatial distribution of up to 40 proteins or RNA molecules at $1 \mu \mathrm{m}$ pixel size $[5,28]$.

\subsection{Multi-channel image visualization with napari}

Visual inspection of imaging data is key to bioimage analysis, and specialized software is required for multi-channel image visualization [29, 30]. The recently developed multi-dimensional image viewer napari enables the fast and interactive visualization of multi-channel images, supported by a growing community of developers [31]. Through plugins written in Python, napari can be extended to load image data from a variety of multiplexed imaging platforms.

Multiplexed imaging often generates complex raw data that can be challenging to visualize and process. An example for such data is the proprietary MCD file format for IMC: After image acquisition, a single MCD file can hold raw acquisition data for multiple regions of interest, optical images providing a slide level overview of the sample ("panoramas"), and detailed metadata about the experiment. To facilitate IMC data processing, we created readimc, an open-source Python package for extracting the multi-modal (IMC acquisitions, panoramas), multi-region, multi-channel information contained in raw IMC files.

Building on readimc, we developed napari-imc, a modular plugin for loading raw IMC data into napari. Upon opening MCD files, napari-imc displays a graphical user interface for loading panoramas, acquisitions (Figure 2A) and channels (Figure 2B). For each loaded panorama and for each combination of loaded acquisition and channel, napari-imc creates an image layer (Figure $2 \mathrm{C}$ ). In napari, image layers represent single-channel grayscale or color images that can be overlaid in the main panel (Figure 2D). Importantly, all image layers are spatially aligned. Adjusting channel settings (Figure $2 \mathrm{E}$ ) will broadcast the chosen values to the settings of all associated image layers (Figure $2 \mathrm{~F}$ ).

The napari-imc plugin enables the rapid visualization and quality control of raw IMC data, without the need for manual file conversion. Unlike existing software, napari-imc loads multichannel acquisitions and panoramas into a shared coordinate system, allowing for quick spatial orientation with respect to the physical tissue slide. Jointly configuring layers by their channel enables the qualitative comparison of multiple regions of interest. Further, owing to its modular implementation, napari-imc is ready for extension to similar data formats in the future. In summary, using napari-imc, the user can perform a quick, qualitative inspection of multiplexed image acquisitions prior to downstream image processing. 


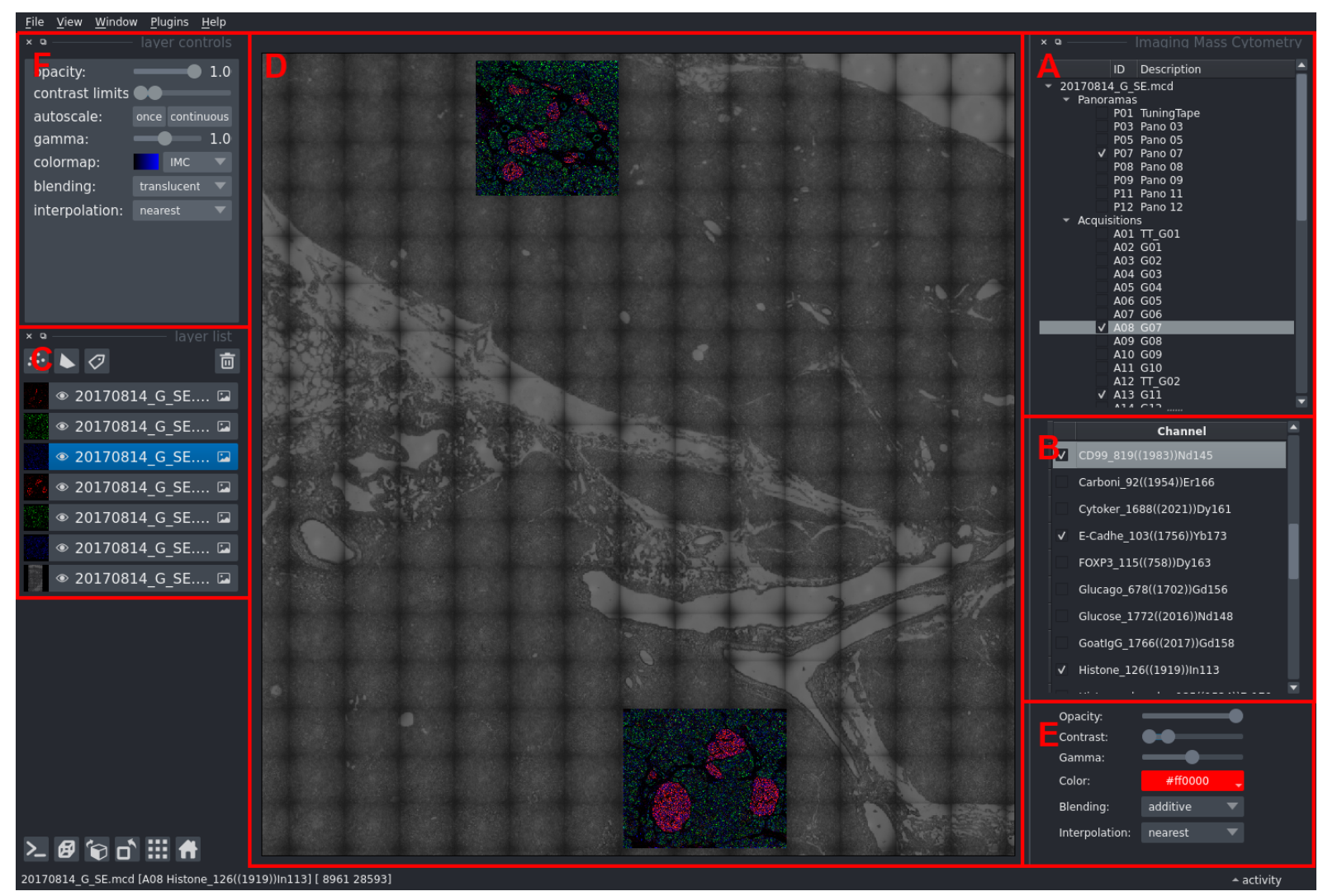

Figure 2: Visualization of raw IMC data using napari-imc. Screenshot of napari-imc visualizing two selected acquisitions from Damond et al. 32. overlaid on one panorama. Image channels corresponding to markers for CD99 (red, cytoplasm), E/P-Cadherin (green, cytoplasm), and Histone H3 (blue, nucleus) are displayed. Labeled boxes (red) indicate GUI elements of napari (C,D,F) and napari$i m c$ (A,B,E): (A) MCD files containing panoramas and multi-channel acquisitions, (B) image channels of all loaded acquisitions, (C) image layers corresponding to loaded panoramas or per-channel images from individual IMC acquisitions, (D) visualization of the image layers, (E) settings of the selected image channel that are broadcast to the properties of associated image layers, $(\mathbf{F})$ properties of the image layers.

\subsection{Multi-channel image processing with steinbock}

Image processing forms the basis for data analysis in any quantitative imaging project. Core tasks of image processing include extraction of images from raw data, segmentation and quantification of spatial entities such as cells, and data export for downstream analysis. Despite their repetitive character, each step requires meticulous quality control of its intermediate outputs, a characteristic often neglected by fully automated image processing pipelines. Further, the individual processing steps must be reproducible, and software must be easy to install and use. To ensure compatibility, all inputs and results must interface with existing tools and approaches.

To facilitate multiplexed image analysis, we developed steinbock, a collection of tools for rapid processing of multi-channel images. The steinbock framework builds on existing approaches, and can be operated through its easy-to-use command-line interface (CLI). Using simple commands, multi-channel images can be processed step by step, offering full control of the individual 


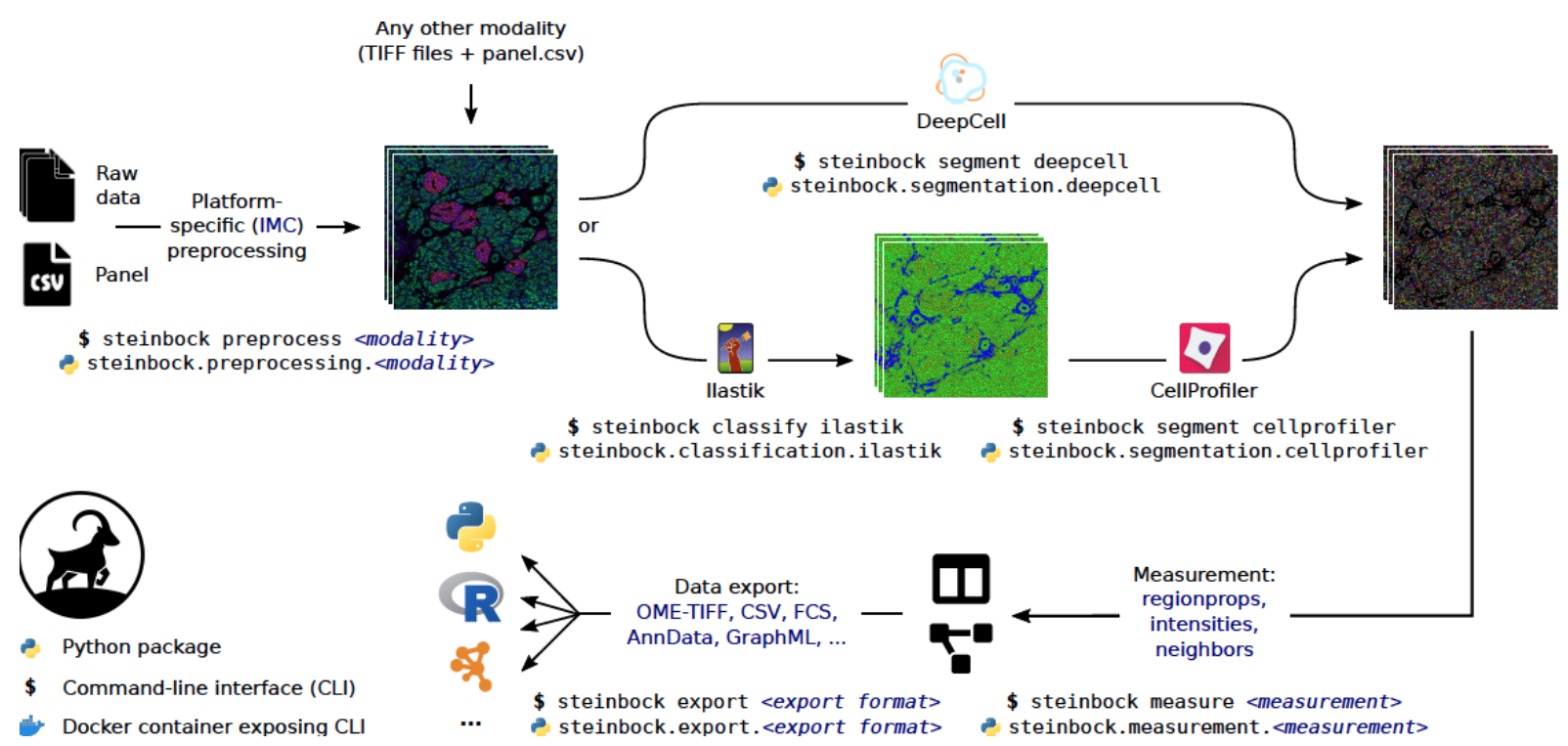

Figure 3: A typical multiplexed image processing workflow using steinbock. Individual steps are indicated as arrows, with corresponding steinbock commands and Python modules shown below. Further parameters and third-party software (bundled in the steinbock Docker container) are overlaid on the arrows. Input and output data are indicated conceptually and described in further detail in Section 2.2

tasks. The framework is distributed as a Docker container that bundles third-party software required for the individual tasks and offers platform-independence and reproducibility. Internally, steinbock is implemented in Python, and the steinbock Python package can be used programmatically.

Both the steinbock CLI and the steinbock Python package are fully documented and have been extensively tested. The steinbock framework integrates with existing software by processing data in standardized formats and its modular open-source implementation enables community-driven development. The tools provided by steinbock can be used to build multiplexed image processing workflows, a typical example is shown in Figure 3.

\subsubsection{Data input and pre-processing}

The steinbock framework operates on multi-channel TIFF images, which can be either provided by the user or extracted from supported raw data formats using steinbock. When starting from supported raw data formats, images are extracted and pre-processed on a pixel level tailored to the specific imaging platform. In the case of IMC, this involves image extraction from raw data using readimc, extraction of metadata, filtering and sorting of image channels, and removal of hot pixels as described in the original IMC Segmentation Pipeline [9]. To enable processing of large images that would otherwise exhaust computational resources, steinbock offers functionality for tiling and stitching images.

In addition to the images, steinbock requires channel-specific metadata (e.g., channel names), 
which can be supplied in CSV format (e.g., a "panel file"). In the case of IMC, such panel files can be automatically generated from the raw data.

\subsubsection{Image segmentation}

After image extraction, image segmentation is performed to define spatial entities such as cells, yielding segmentation masks (e.g., cell masks). Segmentation masks are single-channel images that match the input images in size, with non-zero grayscale values indicating the IDs of segmented objects. The steinbock framework explicitly supports the following supervised image segmentation approaches:

- Random forest-based image segmentation as presented by Zanotelli and Bodenmiller [9]. Briefly, a random forest is trained using Ilastik [7] on randomly extracted image crops and selected image channels to classify pixels as nuclear, cytoplasmic, or background. Employing a customizable CellProfiler [8 pipeline, the probabilities are then thresholded for segmenting nuclei, and nuclei are expanded into cytoplasmic regions to obtain cell masks.

- Deep learning-based image segmentation as presented by Greenwald et al. 15]. Briefly, steinbock first aggregates user-defined image channels to generate two-channel images representing nuclear and cytoplasmic signals. Next, the DeepCell Python package is used to run Mesmer, a deep learning-enabled segmentation algorithm pre-trained on TissueNet [15], to automatically obtain cell masks without any further user input.

\subsubsection{Object quantification}

Following image segmentation, features of the detected objects (e.g., cells) are quantified. The steinbock framework is equipped with functionality for measuring region properties (e.g., area, eccentricity), aggregated marker intensities (e.g., mean, median), and spatial neighbors. The measurement of spatial neighbors yields spatial object graphs, in which nodes correspond to objects, and nodes in spatial proximity are connected by an edge. Distances between objects are computed based on the objects' centroids or borders. These distances are used to construct spatial object graphs by distance thresholding or $k$-nearest neighbor $(k-\mathrm{NN})$ detection. Additionally, steinbock can construct spatial object graphs by the means of morphological dilation ("pixel expansion"). The choice of neighborhood measurement depends on the downstream data analysis approach. For example, pixel expansion is commonly used for neighborhood analysis as presented by Schapiro et al. [16]. 
bioRxiv preprint doi: https://doi.org/10.1101/2021.11.12468357; this version posted November 13,2021 . The copyright holder for this preprint (which was not certified by peer review) is the author/funder, who has granted bioRxiv a license to display the preprint in perpetuity. It is made available under aCC-BY 4.0 International license.

\subsubsection{Data output and export}

All data generated by steinbock are stored in standardized, well-documented file formats that are directly supported by a wide range of third-party software. Specifically, the imcRtools package can be used to load single-cell data generated by steinbock into SingleCellExperiment [25] or SpatialExperiment [33] objects for downstream analysis in $\mathrm{R}$ (described in detail in Section 2.4).

To further facilitate compatibility with downstream analysis, data can additionally be exported to a variety of file formats such as OME-TIFF for images, CSV and FCS for single-cell data, the anndata 34 format for data analysis in Python, and various graph file formats for network analysis using software such as CytoScape [35]. For export to OME-TIFF, steinbock uses xtiff, a Python package we developed for writing multi-channel TIFF stacks.

\subsection{Segmentation quality control with cytomapper}

Visual assessment of image segmentation quality is crucial to avoiding biases in downstream analyses. Visualization is commonly done by outlining segmented objects (e.g., cells) on composite images showing features of interest (e.g., marker proteins). We previously developed the cytomapper R/Bioconductor package 27] to visualize multi-channel images and to map cellular features onto segmentation masks. The cytomapper package supports reading and storage of multi-channel images and segmentation masks including the TIFF files generated by steinbock (Figure 11. Upon data import, multi-channel images can be visualized as composite images with up to six colors (Figure $4 \mathrm{~A}$ ). More importantly in the context of image segmentation, multi-channel images and segmentation masks can be combined to outline segmented cells on composite images (Figure 4B).

A

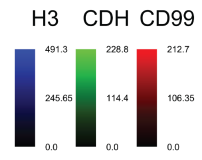

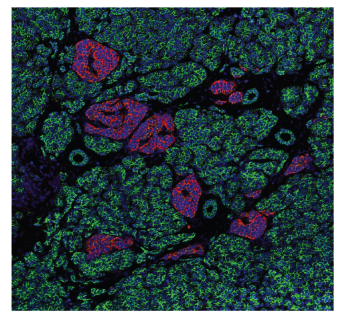

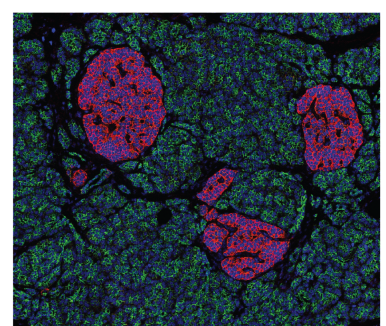

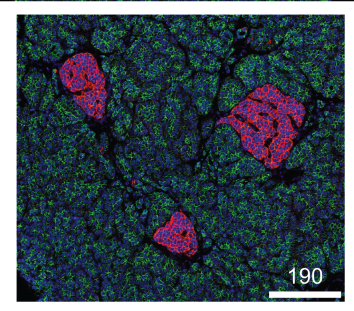

B
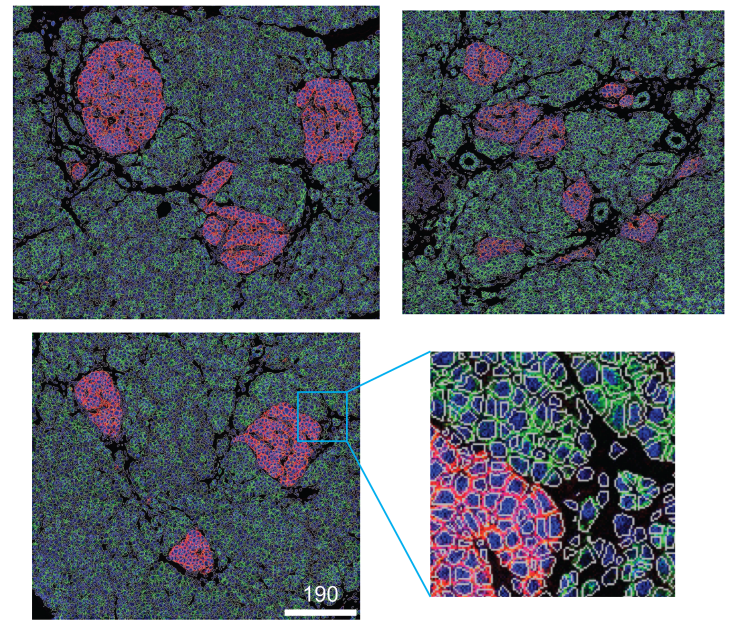

Figure 4: Visualization of multiplexed images and segmentation masks using cytomapper. (A) Composite images showing Histone H3 (H3, blue, nucleus), E/P-cadherin (CDH, green, cytoplasm), and CD99 (red, cytoplasm) expression. (B) Composite images from (A) with segmented cells outlined in white with a zoom plot shown to the right. 


\subsection{Spatial data analysis with imcRtools}

Highly multiplexed imaging generates images from which, after image segmentation, objectspecific measurements can be extracted. Most often, segmented objects represent cells in their tissue context. Common tasks for single-cell data analysis include clustering and cell phenotyping, dimensionality reduction, and differential analysis; standardized methods for these tasks have been previously developed [25]. The imcRtools $\mathrm{R}$ package, available through the Bioconductor project, provides functionality that supports handling of data derived from multiplexed imaging technologies, spatial single-cell analysis, and spatial visualization.

During image pre-processing, steinbock generates text files containing the summarized intensity per cell and channel, properties of the segmented cells (e.g., location, morphology), and spatial object graphs indicating cells in close proximity. The imcRtools package reads the steinbock output and jointly stores single-cell and neighborhood information in a SpatialExperiment or SingleCellExperiment object (Figure 1). These classes directly support single-cell analysis using a variety of analysis packages, including scater [24], scran [23], BayesSpace [26], and cytomapper [27]. The following sections describe functions provided by imcRtools that take a SpatialExperiment or SingleCellExperiment object as standardized input.

\subsubsection{Spatial data visualization}

The visualization of multi-channel images and mapping of cellular features onto segmentation masks can be performed using the cytomapper package [27]. Complementary to this pixel-level visualization strategy, the imcRtools package supports visualizing locations of cells as well as spatial object graphs. The centroids of cells are visualized as points, and lines are drawn between cells in spatial proximity (also referred to as neighboring cells). The lines represent edges in the spatial object graphs computed by steinbock or imcRtools. The imcRtools package allows coloring of cells based on marker expression or cellular metadata (e.g., cell type, morphological features; Figure 5A). The size and shape of points can also be adjusted based on cellular metadata. Lines between neighboring cells can be colored by edge features or cellular metadata associated with the cell from which the edge originates (Figure $5 \mathrm{~B}$ ).

\subsubsection{Spatial data analysis}

Single-cell resolved multiplexed imaging produces data that allows analysis of cells with regards to their spatial location. Over the past five years, spatial data analysis approaches have been developed that yield biologically meaningful insights from highly multiplexed imaging data 4 , 16, 28, 36, 37]. The imcRtools package supports such data analysis approaches in a standardized fashion within the Bioconductor framework.

The imcRtools package can be used to summarize each cell's neighborhood by aggregating either across cellular metadata or the expression of neighboring cells. Cells can now be clustered 
A

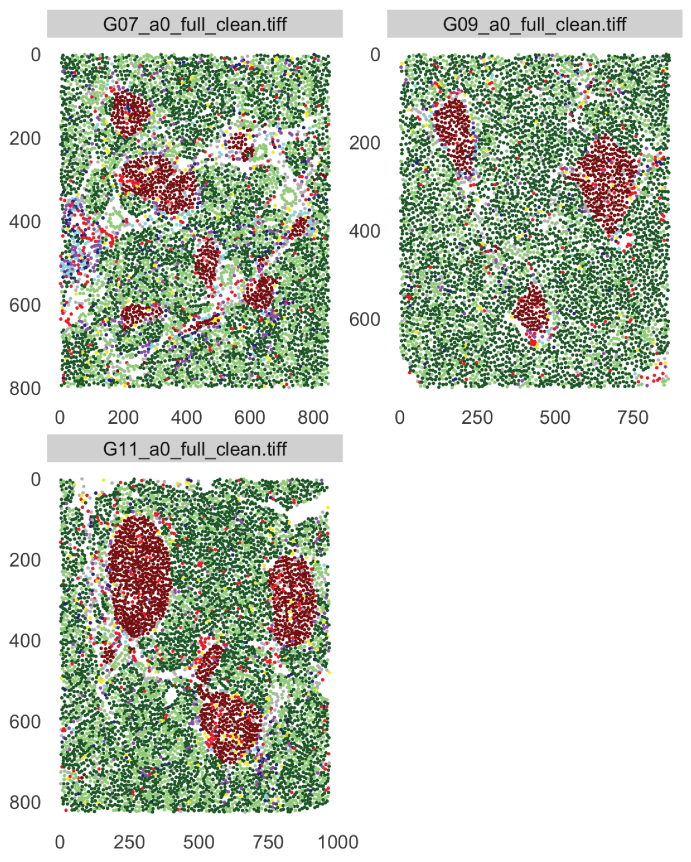

B

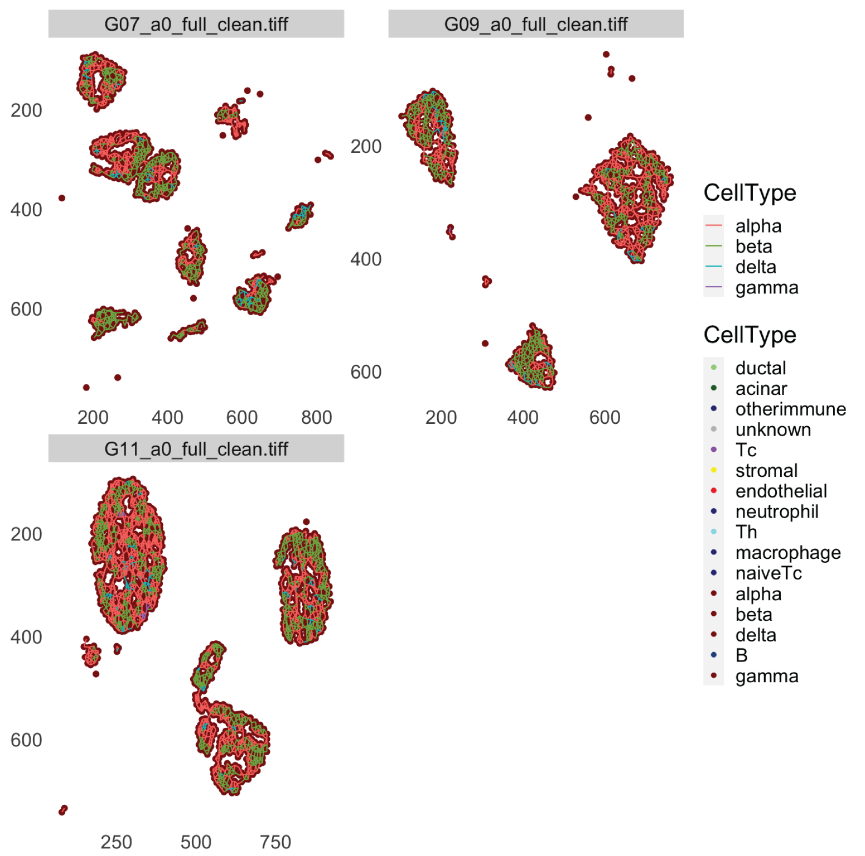

Figure 5: Spatial visualization of cells and their neighborhood information. (A) Points represent the centroids of segmented cells and are colored based on the cells' phenotypes as defined in the original publication 32 . Cells from images displayed in Figure 4 were selected. Islet cells are shown in red; immune cells except $\mathrm{T}$ helper (Th) cells and cytotoxic $\mathrm{T}$ (Tc) cells are shown in dark blue. (B) A $k$-NN spatial object graph was constructed with $k=5$. Only pancreatic islet cells are visualized. As in (A), points represent the centroids of segmented cells and are colored by their phenotype. Cells in spatial proximity are visualized as lines (i.e., edges) between points. Lines are colored by the phenotypes of the cells from which edges originate.

based on aggregated values of neighboring cells, an approach that has been previously proposed by Goltsev et al. [4] and Schürch et al. 36 to characterize cellular neighborhoods. To demonstrate the neighborhood aggregation approach with subsequent clustering, we used imcRtools to analyze a type 1 diabetes dataset provided by Damond et al. [32] as part of the imcdatasets $\mathrm{R} /$ Bioconductor package [38]. For each cell, the proportion of cell types among the 10 nearest neighbors were computed. This information was subsequently used to cluster all cells. The spatial clusters detected in this manner represent different tissue compartments: spatial clusters 1,4 , and 5 contain islet cells, and spatial clusters $3,6,7,8$, and 9 include mainly cells from exocrine tissue. Within the latter, clusters 6,7 , and 9 contain ductal and acinar cells, and clusters 3 and 9 contain a mix of exocrine and stromal cells. Spatial cluster 10 is mainly composed of $\mathrm{T}$ cells and endothelial cells, and cluster 2 involves aggregates of $\mathrm{B}$ and $\mathrm{T}$ cells (Figure 6 $\mathrm{A}$ ). Interestingly, spatial cluster 2 appears in proximity to islets in images obtained from early onset diabetes patients (Figure 6B), possibly indicating immune cell and islet cell interactions.

As an alternative approach, the algorithm developed by Hoch et al. can be used to detect patches [37], where patches are defined as cells of a specific phenotype in spatial proximity. In an optional step, a concave or convex hull is built around the detected patches. This hull can be expanded to include cells in direct vicinity as demonstrated for islet cell types (Figure 6C) 
and immune cell types (Figure6 $\mathrm{D}$ ).

The imcRtools package further allows statistical testing of whether cell types interact more or less frequently than expected by chance using a method implemented by Schapiro et al. as part of the histoCAT software [16. In this context, interacting cells are defined as cells in spatial proximity (i.e., neighboring cells detected by constructing a spatial object graph). First, for each cell of type A the number of neighbors of type B are counted. In each image, this count is averaged in one of three different ways, depending on the question at hand:

1. The count is divided by the total number of cells of type A. The final count can be interpreted as "How many neighbors of type B does a cell of type A have on average?".

2. The count is divided by the number of cells of type A that have at least one neighbor of type B. The final count can be interpreted as "How many neighbors of type B has a cell of type A on average, given it has at least one neighbor of type B?"

3. For each cell, the count is binarized to 0 (less than a specified number of neighbors of type $\mathrm{B})$ or 1 (more than or equal to a specified number of neighbors of type B). The binarized counts are averaged across all cells of type A. The final count can be interpreted as "What fraction of cells of type A have at least a given number of neighbors of type B?". This counting strategy was proposed by Schulz et al. 28.

Next, the computed count is compared to an empirical null distribution of interaction counts. To derive such a distribution, cell labels are randomized a number of times, and for each iteration the interaction count is computed. Statistical inference is performed by comparing the actual interaction count to the empirical null distribution.

As an example, we analyzed the type 1 diabetes data reported by Damond et al. 32 . For all three example images, we observed that $\alpha, \beta$, and $\delta$ cells interact more often than expected when cell types are randomly distributed across the images (Figure $6 \mathrm{E}$ ). This result was expected, because $\alpha, \beta$, and $\delta$ cells are locally confined in pancreatic islets. In addition, we observed increased interactions between naïve cytotoxic $\mathrm{T}$ cells (naïve Tc), cytotoxic $\mathrm{T}$ cells $(\mathrm{Tc}$ ) and helper $\mathrm{T}$ cells (Th) (Figure 6E).

As these examples demonstrate, the imcRtools package provides functionality to (i) handle data extracted from multiplexed images, (ii) visualize cellular information in a spatial manner and (iii) perform spatial data analysis to detect cell aggregates and enriched interactions between cell types. Building upon standardized data classes, the imcRtools package connects bioimage processing as performed using steinbock with downstream analyses supported by the Bioconductor project. 
bioRxiv preprint doi: https://doi. org/10.1101/2021.11.12 468357; this version posted November 13, 2021. The copyright holder for this preprint (which was not certified by peer review) is the author/funder, who has granted bioRxiv a license to display the preprint in perpetuity. It is made available under aCC-BY 4.0 International license.

A

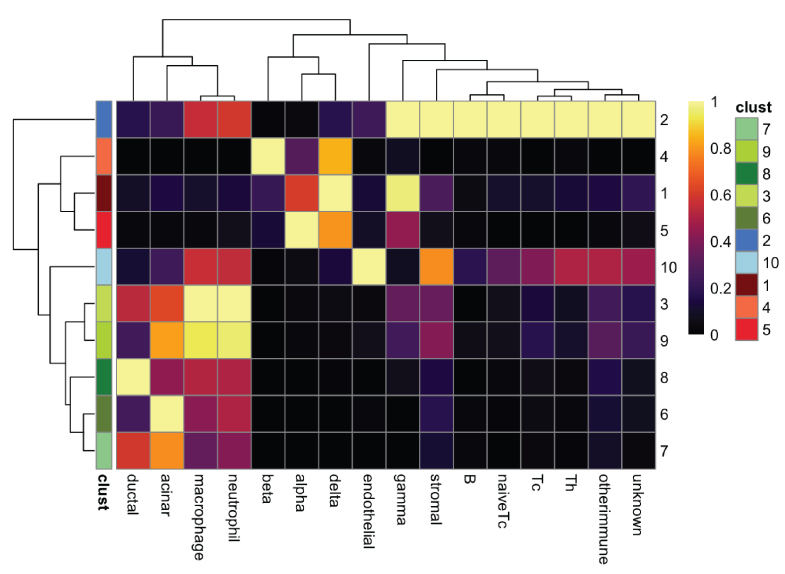

C

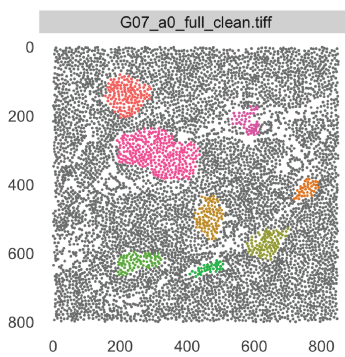

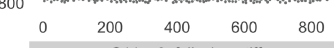

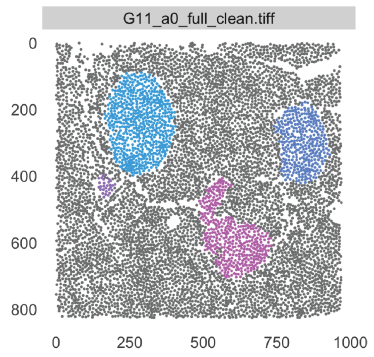

B

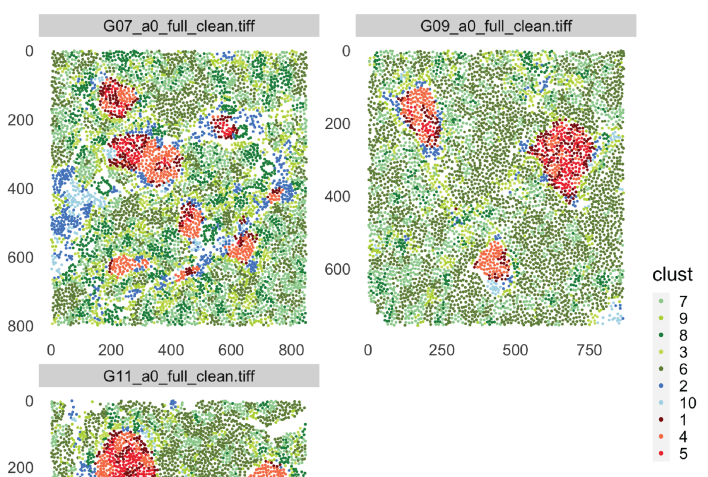

D
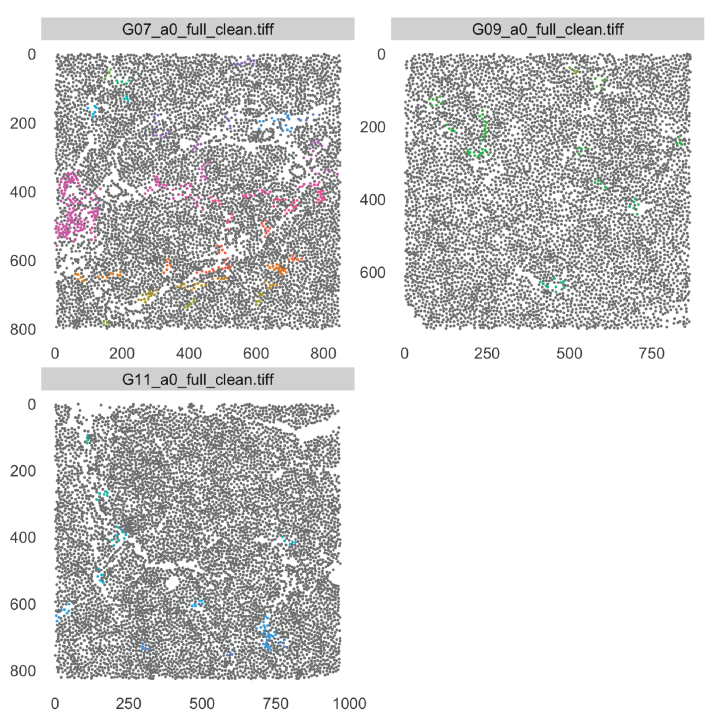

E

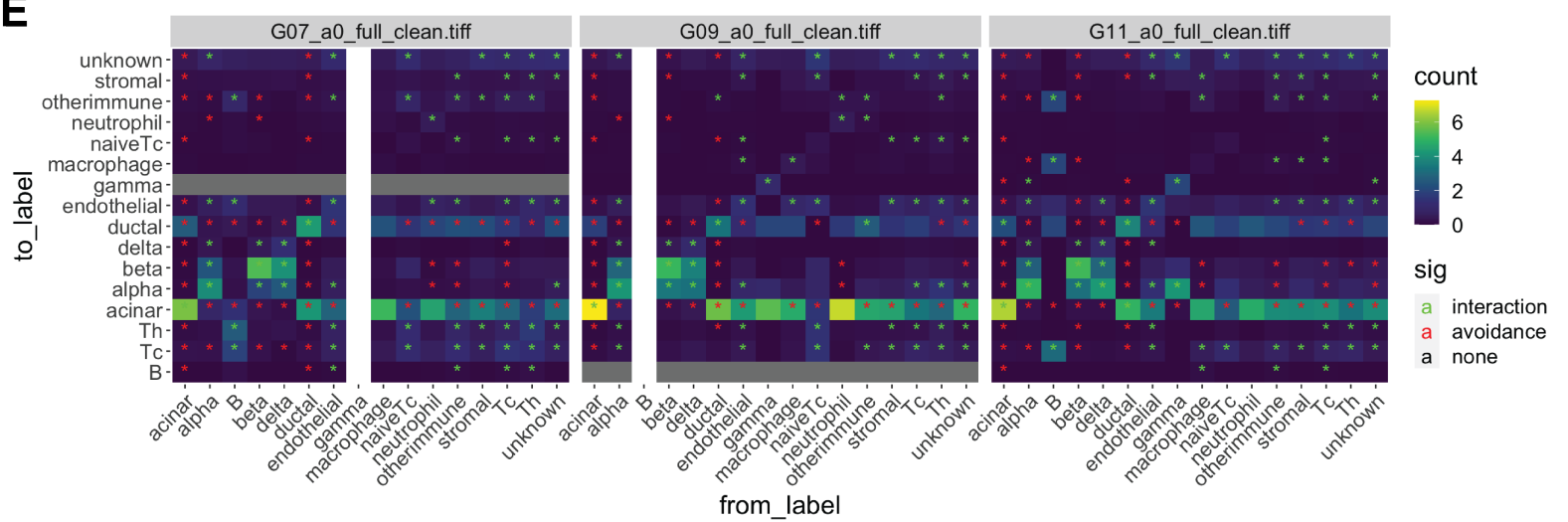

Figure 6: Spatial analysis strategies supported by imcRtools. Figure legend on next page. 
Figure 6: Spatial analysis strategies supported by imcRtools. (A) Cells of the type 1 diabetes dataset generated by Damond et al. 32 were clustered based on cell type proportions among their 10 nearest neighbors. Cell type proportions within each spatial cluster (rows, colored by cluster) are shown as a heatmap. Columns are rescaled between 0 and 1. (B) Points represent the centroids of segmented cells and their color indicates spatial clusters. Cells from images displayed in Figure 4 were selected. (C) $\alpha, \beta, \delta$, and $\gamma$ cells were selected to detect connected patches of islet cells and patches were expanded by $10 \mu \mathrm{m}$. Colored points represent cells within islet patches and grey points indicate cells outside islet patches. (D) Immune cells were selected to detect immune cell aggregates. Colored points represent cells within immune patches and grey points indicate cells outside immune patches. (E) The interaction count between pairs of cell types visualized in form of one heatmap per image. Cell types that interact more often than expected by chance are indicated by a green star (interaction). Cell types that interact less often are indicated by a red star (avoidance). Statistical significance was defined at an empirical p-value threshold of 0.01 .

\section{Discussion}

Here, we present a modular workflow for analyzing highly multiplexed imaging data, introducing the steinbock framework for multi-channel data processing and imcRtools for spatial data analysis. The workflow standardizes common processing and data analysis tasks and integrates bioimage and single-cell analysis in a user-friendly fashion. Due to its modular structure, the workflow is easily extendable to additional image processing steps and new approaches for singlecell and spatial analysis.

Starting from multi-channel TIFF files or raw data from supported imaging platforms, the steinbock framework extracts data for downstream analysis. Depending on the imaging platform, additional pre-processing, such as the alignment of consecutive images for cyclic immunofluorescence modalities [1, 2], may be required before steinbock is run. For IMC, however, steinbock can be directly applied to raw acquisition data, and it performs established pre-processing routines [9. Explicit support for other imaging platforms may be added in the future. Furthermore, processing of large images is enabled by image tiling/stitching. As with all bioimaging approaches, meticulous quality control should be performed at each step of the image processing workflow, including careful inspection of the raw imaging data using tools such as napari [31].

A core task of multi-channel image processing is the segmentation of spatial entities such as cells. The steinbock framework currently supports two previously published image segmentation approaches [9, 15]. The first is the random forest-based approach [9], which is highly customizable, allows for dataset-specific tuning, and has been applied in several multiplexed imaging applications [28, 32, 39]. By adapting the provided Ilastik [7] and CellProfiler [8] pipelines, this approach enables segmentation of arbitrary spatial entities such as tumor/stroma regions or pancreatic islets. However, the random forest classifier needs to be manually trained for each dataset and is limited in its applicability to other projects. The second approach is based on deep learning [15], which builds on existing annotations [15] and can be used to rapidly segment 
cells or nuclei without the need for manually training a classifier. Irrespective of the chosen approach, the validity of the obtained segmentation masks always needs to be verified using tools such as cytomapper [27]. Further approaches for multi-channel image processing may be added in the future.

Unlike most existing software, steinbock was not implemented as a pipeline, but as a collection of independent, easy-to-use tools. Although these tools can be employed in streamlined pipelines, they are intended to be used in step-by-step workflows that offer full control of the individual tasks. This is reflected in the design principles underlying the steinbock framework, which focus on usability and interoperability with external tools. By operating on standardized data formats, steinbock integrates with the growing landscape of multiplexed imaging software and facilitates exploratory bioimage analysis.

To bridge bioimage processing, segmentation and feature extraction with downstream analysis strategies commonly performed in $\mathrm{R}$, we developed the imcRtools package. The imcRtools package offers analysis strategies applicable to single-cell data extracted from most multiplexed imaging technologies [1 6] and can read in data generated by steinbock. It also supports spatial analysis and visualization of single-cell data derived from spatial transcriptomic technologies such as MERFISH [40] and seqFISH [41. Furthermore, the imcRtools package contains functionality specifically for handling and processing of IMC data. These include reading in raw IMC data into CytoImageList objects for visualization with cytomapper or into SingleCellExperiment objects to perform channel spillover correction using the CATALYST R/Bioconductor package 42 .

As part of the Bioconductor project 22] building upon the SingleCellExperiment 25] and SpatialExperiment [33] data classes, the imcRtools package fully integrates with a variety of single-cell and spatial analysis approaches and tools. It furthermore standardizes analysis approaches that were previously developed for highly multiplexed imaging data [4, 16, 28, 36, 37] and therefore complements other spatial data analysis tools such as giotto 21 and spatial clustering approaches including BayesSpace [26] and lisaClust [43]. As highly multiplexed imaging technologies and new ways of interpreting spatially annotated data develop, the imcRtools package will provide a platform for implementing emerging data analysis strategies. 


\section{Methods}

\subsection{Example data}

To highlight the functionality of the imcRtools and cytomapper R/Bioconductor packages, an IMC dataset containing pancreatic islets of healthy and type 1 diabetes patients was reanalyzed [32. The data are available in the imcdatasets $\mathrm{R} /$ Bioconductor package [38] (version 1.2.0) under the accessor: DamondPancreas2019Data.

\subsection{Multi-channel image visualization}

Raw IMC data from Damond et al. [32] was visualized using napari 0.4.11 and napari-imc 0.6.2 for illustration purposes (Figures 2, 3). Two acquisitions in spatial proximity were selected and overlaid on the corresponding panorama (Figure 2), matching the acquisitions in downstream analyses. Channels were configured to match Figure 4, applying the same channel-specific settings to all image layers.

\subsection{Multi-channel image processing}

The steinbock framework was implemented in the steinbock Python package (version 0.10.0), with Python modules corresponding to individual image processing tasks and including a commandline interface (CLI). The steinbock Docker container bundles the steinbock Python package with third-party software required for the individual tasks and exposes the steinbock CLI as Docker entrypoint.

The pre-processing module of steinbock currently supports reading raw IMC data using readimc 0.4.2 (developed in-house). Random forest-based image segmentation was implemented using Ilastik 1.3.3post3 [7] and CellProfiler 4.2.1 [8], and HDF5 files are used for loading multichannel images in Ilastik. Deep learning-based image segmentation was implemented using DeepCell 0.11.0 15, currently supporting Mesmer 15] for nuclear and cell segmentation. Further details on object quantification, data output, and data export can be found in the online documentation.

\subsection{Segmentation quality control}

\section{Image visualization with cytomapper}

All $\mathrm{R}$ based data analyses was performed using $\mathrm{R}$ version 4.1.2.

The cytomapper package version 1.6.0 was used to generate composite images and to overlay segmentation masks on composite images using the plotPixels function to generate Figure 4 
The three largest images considering the number of pixels were selected from the dataset. The channel contrast was increased by scaling the intensities with a factor of 10 for Histone H3, 5 for E-/P-cadherin and 5 for CD99.

\subsection{Spatial data analysis}

\section{Reading in the steinbock data}

The imcRtools package provides the read_steinbock function that reads in the summarized intensities per cell and channel, properties of the segmented cells (e.g., location, morphology), and spatial object graphs indicating cells in close proximity. These information are stored in either a SpatialExperiment [33] or a SingleCellExperiment [25] object. The cell intensity data are stored in the counts assay slot, object properties are stored in the colData slot, and the spatial interaction graphs are stored in a colPair slot. In the case of the SpatialExperiment object, spatial coordinates are stored in the spatialCoords slot, and spatial coordinates in a SingleCellExperiment are stored in the colData slot.

The imcRtools package contains functionality applicable across different multiplexed imaging technologies. In addition, imcRtools further supports IMC-specific data handling. Raw IMC data can be read into CytoImageList objects for visualization with cytomapper. Additionally, raw .txt files from control acquisitions can be read into a SingleCellExperiment object for spillover estimation using CATALYST [42].

\section{Spatial graph construction and visualization}

The graph construction, spatial visualization, and spatial analysis presented here were performed using imcRtools version 1.0.0. To visualize the location of cells and their interactions in Figure 5. a $k$-NN graph based on the cells' centroids was constructed for $k=5$ using the buildSpatialGraph function from the imcRtools package. The buildSpatialGraph function additionally supports constructing spatial object graphs based on distance thresholding or Delaunay triangulation to identify cells in close spatial proximity. The plotSpatial function was used to visualize the cells' locations as points and to draw lines between cells if they were detected as neighbors using the 5-nearest neighbor graph construction approach.

\section{Spatial data analysis}

For the unsupervised spatial clustering analysis presented in Figure 6 $\mathrm{A}, \mathrm{B}$, a $k$-NN graph based on the cells' centroids was constructed for $k=10$ using the buildSpatialGraph function. The aggregateNeighbors function from the imcRtools package was used to calculate the proportions of cell types among the 10-nearest neighbors. Cells were further clustered based on these proportions using k-means clustering with $k=10$. For each spatial cluster, the number of cells of 
each cell type was divided by the cluster size. Next, fraction values were rescaled between 0 and 1 per cell type (Figure6A). Instead of aggregating the cell type, the mean or median expression across all neighboring cells can be computed using the aggregateNeighbors function.

Figure 6 6 ,D present an alternative approach to detect spatial aggregates (i.e., patches) of cells. The imcRtools package provides the patchDetection function, which detects patches of neighboring cells of a pre-defined type. To detect the pancreatic islets (Figure6 $6 \mathrm{C}$ ), a 10-nearest neighbor graph was constructed using the cells' centroids, and neighboring $\alpha, \beta, \delta$ and $\gamma$ cells were selected to detect connected patches. A concave hull was constructed around individual patches, and the hull was expanded by $10 \mu \mathrm{m}$ to include cells in direct vicinity. Next, to detect patches that define interacting immune cells (Figure 6D), the 10-nearest neighbor graph was used to find patches of neighboring Tc, Th, naïve Tc, neutrophiles, macrophages, other immune cells and B cells. Patches with at least 4 cells were considered to represent aggregations of immune cells.

To test whether cell types interact more or less frequently compared to a random distribution, the testInteractions function available in the imcRtools package was used. First, a 10-nearest neighbor graph was constructed to detect neighboring cells. For each cell type pair "A" and "B", the overall interaction count was divided by the number of cells of type "A" (Figure 6E). The count is compared to the random distribution of interaction counts, which was derived by permuting cell type labels 1000 times. Statistical significance for avoidance was defined if more than 990 iterations of random permutations produced larger counts. Statistical significance for association was defined if more than 990 iterations of random permutations produced smaller counts.

\section{Software and code availability}

The readimc Python package is available from https://github.com/BodenmillerGroup/readimc and installable via pip. Documentation is available at https://bodenmillergroup.github.io/ readimc.

The napari-imc Python package/napari plugin is available from https://github.com/BodenmillerGroup/ napari-imc and installable via pip, from within napari or from https://www.napari-hub. org.

The steinbock framework is available from/https://github.com/BodenmillerGroup/steinbock. The steinbock Python package is installable via pip. The steinbock Docker container can be obtained via Docker from ghcr.io/bodenmillergroup/steinbock. Documentation is available at https://bodenmillergroup.github.io/steinbock

The xtiff Python package is available from https://github.com/BodenmillerGroup/xtiff and installable via pip. 
The imcRtools development version is available from https://github.com/BodenmillerGroup/ imcRtools. The release version is installable via Bioconductor: https://bioconductor.org/ packages/imcRtools. Documentation is available at https://bodenmillergroup.github.io/ imcRtools/.

The images and masks shown for illustration purposes in Figure 3 were taken from publicly available resources [32] and visualized using Python and napari. For convenience of the reader, the corresponding Jupyter notebook for loading multi-channel images, cell masks and cell outlines in napari is available from https://github.com/BodenmillerGroup/IMCDataAnalysis/ tree/biorxiv_submission/code/mask_overlay.

The $\mathrm{R}$ analysis code used to highlight the functionality of the cytomapper and imcRtools packages is available from https://github.com/BodenmillerGroup/IMCDataAnalysis/tree/ biorxiv_submission.

\section{Contributions}

J.W. developed and maintains the steinbock framework, napari-imc, readimc and xtiff. N.E. developed and maintains the imcRtools R package. J.W. and N.E. wrote the manuscript. B.B. carried senior authorship responsibility. All authors approve the manuscript.

\section{Acknowledgments}

We want to thank Jana R. Fischer, Daniel Schulz and Tobias Hoch for code contributions to the imcRtools package. Special thanks go to Vito R.T. Zanotelli for code contributions to the imcRtools package and for developing the conceptual underpinning of the IMC Segmentation Pipeline. We thank Nicolas Damond for providing the raw data associated to the type 1 diabetes dataset.

We also thank Daniel Schulz, Tess Brodie and Joseena Iype for critically reading and giving feedback on the manuscript.

J.W. was funded by the CRUK IMAXT Grand Challenge. B.B. was supported by a SNSF R'Equip grant, a SNSF Assistant Professorship grant, the SystemsX Transfer Project "Friends and Foes," the SystemX grants Metastasix and PhosphoNEtX, a NIH grant (UC4 DK108132), the CRUK IMAXT Grand Challenge, and by the European Research Council (ERC) under the European Union's Seventh Framework Program (FP/2007-2013)/ERC grant agreement no. 336921. N.E. was funded by the European Union's Horizon 2020 research and innovation program under Marie Sklodowska-Curie Actions grant agreement No 892225. 


\section{References}

[1] Jia-Ren Lin et al. "Highly multiplexed immunofluorescence imaging of human tissues and tumors using t-CyCIF and conventional optical microscopes". In: eLife 7 (2018), e31657. DOI: $10.7554 /$ eLife.31657.

[2] Gabriele Gut, Markus D Herrmann, and Lucas Pelkmans. "Multiplexed protein maps link subcellular organization to cellular states". In: Science 361 (2018), eaar7042. DoI: 10.1126/science. aar7042.

[3] Sinem K Saka et al. "Immuno-SABER enables highly multiplexed and amplified protein imaging in tissues". In: Nature Biotechnology 37 (2019), pp. 1080-1090. DOI: 10.1038/ s41587-019-0207-y.

[4] Yury Goltsev et al. "Deep Profiling of Mouse Splenic Architecture with CODEX Multiplexed Imaging". In: Cell 174 (2018), pp. 968-981. DOI: 10.1016/j.cell.2018.07.010.

[5] Charlotte Giesen et al. "Highly multiplexed imaging of tumor tissues with subcellular resolution by mass cytometry". In: Nature Methods 11 (2014), pp. 417-422. DOI: 10 . 1038/nmeth.2869.

[6] Michael Angelo et al. "Multiplexed ion beam imaging of human breast tumors". In: Nature Medicine 20 (2014), pp. 436-442. DOI: 10.1038/nm.3488.

[7] Stuart Berg et al. "ilastik: interactive machine learning for (bio)image analysis". In: Nature Methods (2019). DOI: 10.1038/s41592-019-0582-9.

[8] Claire McQuin et al. "CellProfiler 3.0: Next-generation image processing for biology". In: PLOS Biology 16 (2018). Ed. by Tom Misteli, e2005970. DOI: 10.1371/journal .pbio. 2005970.

[9] Vito RT Zanotelli and Bernd Bodenmiller. ImcSegmentationPipeline: A pixelclassification based multiplexed image segmentation pipeline. Version 0.9. Sept. 2017. DOI: 10.5281/ zenodo.3841961.

[10] Harshil Patel et al. nf-core/imcyto: nf-core/imcyto v1.0.0 - Platinum Panda. 2020. DoI: 10.5281/ZENODO .3865430.

[11] Denis Schapiro et al. "MCMICRO: A scalable, modular image-processing pipeline for multiplexed tissue imaging". In: bioRxiv (2021). DOI: 10.1101/2021.03.15.435473

[12] Xu Xiao et al. "Dice-XMBD: Deep learning-based cell segmentation for imaging mass cytometry". In: bioRxiv (2021). DOI: 10.1101/2021.06.05.447183.

[13] Uwe Schmidt et al. "Cell Detection with Star-Convex Polygons". In: 2018, pp. 265-273. DOI: $10.1007 / 978-3-030-00934-2 \_30$.

[14] Carsen Stringer et al. "Cellpose: a generalist algorithm for cellular segmentation". In: Nature Methods 18 (2020), pp. 100-106. DOI: 10.1038/s41592-020-01018-x.

[15] Noah F. Greenwald et al. "Whole-cell segmentation of tissue images with human-level performance using large-scale data annotation and deep learning". In: bioRxiv (2021). DOI: $10.1101 / 2021.03 .01 .431313$ 
[16] Denis Schapiro et al. "histoCAT: analysis of cell phenotypes and interactions in multiplex image cytometry data". In: Nature Methods 14 (2017), pp. 873-876. Dor: 10.1038/nmeth. 4391

[17] Caleb R Stoltzfus et al. "CytoMAP: A Spatial Analysis Toolbox Reveals Features of Myeloid Cell Organization in Lymphoid Tissues". In: Cell Reports 31 (2020), p. 107523. DOI: $10.1016 /$ j.celrep.2020.107523

[18] Eric Czech et al. "Cytokit: a single-cell analysis toolkit for high dimensional fluorescent microscopy imaging". In: BMC Bioinformatics 20 (2019). DOI: 10.1186/s12859-0193055-3.

[19] Antonios Somarakis et al. "ImaCytE: Visual Exploration of Cellular Micro-Environments for Imaging Mass Cytometry Data". In: IEEE Transactions on Visualization and Computer Graphics 27 (2021), pp. 98-110. DOI: 10.1109/TVCG.2019.2931299.

[20] Giovanni Palla et al. "Squidpy: a scalable framework for spatial single cell analysis". In: bioRxiv (2021). DOI: 10.1101/2021.02.19.431994.

[21] Ruben Dries et al. "Giotto: a toolbox for integrative analysis and visualization of spatial expression data". In: Genome Biology 22 (2021). DOI: 10.1186/s13059-021-02286-2.

[22] Robert C Gentleman et al. "Bioconductor: open software development for computational biology and bioinformatics". In: Genome Biology 5 (2004), R80. DoI: 10.1186/gb-20045-10-r80.

[23] Aaron T L Lun, Davis J McCarthy, and John C Marioni. "A step-by-step workflow for low-level analysis of single-cell RNA-seq data with Bioconductor". In: F1000Research 5 (2016), p. 2122. DOI: 10.12688/f1000research.9501.2.

[24] Davis J McCarthy et al. "Scater: pre-processing, quality control, normalization and visualization of single-cell RNA-seq data in R". In: Bioinformatics 33 (2017), pp. 1179-1186. DOI: 10.1093 /bioinformatics/btw777.

[25] Robert A Amezquita et al. "Orchestrating single-cell analysis with Bioconductor". In: Nature Methods 17 (2020), pp. 137-145. DOI: 10.1038/s41592-019-0654-x.

[26] Edward Zhao et al. "Spatial transcriptomics at subspot resolution with BayesSpace". In: Nature Biotechnology (2021). DOI: 10.1038/s41587-021-00935-2.

[27] Nils Eling et al. "cytomapper: an R/Bioconductor package for visualization of highly multiplexed imaging data". In: Bioinformatics 36 (2020), pp. 5706-5708. DOI: 10.1093/ bioinformatics/btaa1061.

[28] Daniel Schulz et al. "Simultaneous Multiplexed Imaging of mRNA and Proteins with Subcellular Resolution in Breast Cancer Tissue Samples by Mass Cytometry". In: Cell Systems 6 (2018), pp. 25-36. DOI: 10.1016/j.cels.2017.12.001.

[29] Johannes Schindelin et al. "Fiji: an open-source platform for biological-image analysis". In: Nature Methods 9 (2012), pp. 676-682. DOI: 10.1038/nmeth.2019. 
[30] Peter Bankhead et al. "QuPath: Open source software for digital pathology image analysis". In: Scientific Reports 7 (2017). DOI: 10.1038/s41598-017-17204-5.

[31] Nicholas Sofroniew et al. napari/napari: 0.4.11. 2021. DOI: 10.5281/ZENOD0.5399494.

[32] Nicolas Damond et al. "A Map of Human Type 1 Diabetes Progression by Imaging Mass Cytometry". In: Cell Metabolism 29 (2019), 755-768.e5. DoI: 10.1016/j.cmet.2018.11. 01 .

[33] Dario Righelli et al. "SpatialExperiment: infrastructure for spatially resolved transcriptomics data in R using Bioconductor". In: bioRxiv (2021). DOI: 10.1101/2021.01.27. 428431 .

[34] F. Alexander Wolf, Philipp Angerer, and Fabian J. Theis. "SCANPY: large-scale singlecell gene expression data analysis". In: Genome Biology 19 (2018). DOI: 10.1186/s13059017-1382-0.

[35] Paul Shannon et al. "Cytoscape: A Software Environment for Integrated Models of Biomolecular Interaction Networks". In: Genome Research 13 (2003), pp. 2498-2504. DOI: 10.1101/ gr.1239303

[36] Christian M Schürch et al. "Coordinated Cellular Neighborhoods Orchestrate Antitumoral Immunity at the Colorectal Cancer Invasive Front”. In: Cell 182 (2020), pp. 1341-1359. DOI: $10.1016 / \mathrm{j}$. cell.2020.07.005.

[37] Tobias Hoch et al. "Multiplexed Imaging Mass Cytometry of Chemokine Milieus in Metastatic Melanoma Characterizes Features of Response to Immunotherapy". In: bioRxiv (2021). DOI: $10.1101 / 2021.07 .29 .454093$.

[38] Nicolas Damond. imcdatasets: Collection of publicly available imaging mass cytometry (IMC) datasets. Version 1.0.1. DOI: 10.18129/B9.bioc.imcdatasets.

[39] Hartland W. Jackson et al. "The single-cell pathology landscape of breast cancer". In: Nature 578.7796 (2020), pp. 615-620. DOI: 10.1038/s41586-019-1876-x.

[40] Kok Hao Chen et al. "Spatially resolved, highly multiplexed RNA profiling in single cells". In: Science 348 (2015). DOI: 10.1126/science.aaa6090.

[41] Eric Lubeck et al. "Single-cell in situ RNA profiling by sequential hybridization". In: Nature Methods 11 (2014), pp. 360-361. DOI: 10.1126/science.aaa6090.

[42] Stéphane Chevrier et al. "Compensation of Signal Spillover in Suspension and Imaging Mass Cytometry". In: Cell Systems 6 (2018), pp. 612-620. DOI: 10.1016/j.cels.2018. 02.010 .

[43] Ellis Patrick et al. "Spatial analysis for highly multiplexed imaging data to identify tissue microenvironments". In: bioRxiv (2021). DOI: 10.1101/2021.08.16.456469. 